\title{
Modelling DW-MRI data from primary and metastatic ovarian tumours
}

\author{
Jessica M. Winfield • Nandita M. deSouza • Andrew N. Priest • Jennifer C. Wakefield • \\ Charlotte Hodgkin • Susan Freeman • Matthew R. Orton • David J. Collins
}

Received: 29 August 2014 /Revised: 13 November 2014 / Accepted: 16 December 2014 / Published online: 21 January 2015

(C) The Author(s) 2015. This article is published with open access at Springerlink.com

\begin{abstract}
Objectives To assess goodness-of-fit and repeatability of mono-exponential, stretched exponential and bi-exponential models of diffusion-weighted MRI (DW-MRI) data in primary and metastatic ovarian cancer.

Methods Thirty-nine primary and metastatic lesions from thirty-one patients with stage III or IV ovarian cancer were examined before and after chemotherapy using DW-MRI with ten diffusion-weightings. The data were fitted with (a) a monoexponential model to give the apparent diffusion coefficient (ADC), (b) a stretched exponential model to give the distributed diffusion coefficient (DDC) and stretching parameter $(\alpha)$, and (c) a bi-exponential model to give the diffusion coefficient (D), perfusion fraction $(f)$ and pseudodiffusion coefficient $\left(\mathrm{D}^{*}\right)$. Results Coefficients of variation, established from repeated baseline measurements, were: ADC $3.1 \%$, DDC $4.3 \%, \alpha$ $7.0 \%$, D $13.2 \%, f 44.0 \%$, D* $165.1 \%$. The bi-exponential model was unsuitable in these data owing to poor repeatability. After excluding the bi-exponential model, analysis using Akaike
\end{abstract}

J. M. Winfield • N. M. deSouza • J. C. Wakefield • M. R. Orton • D. J. Collins

CRUK and EPSRC Cancer Imaging Centre, Division of Radiotherapy and Imaging, Institute of Cancer Research, 15 Cotswold Road, Sutton, Surrey SM2 5NG, UK

J. M. Winfield $\cdot$ N. M. deSouza $\cdot$ D. J. Collins

Royal Marsden NHS Foundation Trust, Downs Road, Sutton, Surrey SM2 5PT, UK

\section{A. N. Priest $\cdot$ C. Hodgkin $\cdot$ S. Freeman}

Department of Radiology, Addenbrooke's Hospital, University of Cambridge, Hills Road, Box 218, Level 5, Cambridge CB2 0QQ, UK

J. M. Winfield $(\bowtie)$

MRI Unit, Institute of Cancer Research and Royal Marsden Hospital, Downs Road, Sutton, Surrey SM2 5PT, UK

e-mail: jessica.winfield@icr.ac.uk
Information Criteria showed that the stretched exponential model provided the better fit to the majority of pixels in $64 \%$ of lesions. Conclusions The stretched exponential model provides the optimal fit to DW-MRI data from ovarian, omental and peritoneal lesions and lymph nodes in pre-treatment and posttreatment measurements with good repeatability.

Key points

- DW-MRI data in ovarian cancer show deviation from monoexponential behaviour

- Parameters derived from the stretched exponential model showed good repeatability (CV $7 \%$ )

- The bi-exponential model was unsuitable because of poor parameter repeatability

- The stretched exponential model showed comparable repeatability to the mono-exponential model

- The extra parameter $(\alpha)$ provides scope for investigation of heterogeneity or response

Keywords Magnetic resonance imaging · Diffusion · Ovary · Neoplasms $\cdot$ Metastasis

$\begin{array}{ll}\text { Abbreviations } \\ \text { DW-MRI } & \text { Diffusion-weighted magnetic resonance imaging } \\ \text { ADC } & \text { Apparent diffusion coefficient } \\ \text { DDC } & \text { Distributed diffusion coefficient } \\ \text { IVIM } & \text { Intravoxel incoherent motion } \\ \text { ROI } & \text { Region of interest } \\ \text { SNR } & \text { Signal-to-noise ratio } \\ \text { AIC } & \text { Akaike information criterion } \\ \text { CV } & \text { Coefficient of variation }\end{array}$

\section{Introduction}

Diffusion-weighted magnetic resonance imaging (DW-MRI) depicts solid tumours with high contrast because they exhibit 
increased restriction of water diffusion compared with many normal tissues. DW-MRI has been used in patients with ovarian cancer for localisation of disease, monitoring response to treatment, and in the investigation of recurrence [1-6]. DWMRI may also provide a quantitative assessment of response to chemotherapy because an increase in the apparent diffusion coefficient (ADC) after chemotherapy, measured over the entire disease burden, has been shown to be indicative of response to treatment [7, 8]. Estimates of ADC are derived from fitting a mono-exponential function to the signal (S) as described by Eq. 1 .

$S(b)=S_{0} \exp (-b \cdot A D C)$

DW-MRI may be valuable in assessing inter-lesion heterogeneity, for example, lower baseline ADCs have been reported in peritoneal deposits when compared with ovarian or omental lesions [8] and post-treatment changes may also vary by disease site [9]. However, it has previously been shown that the DW-MRI signal in primary and metastatic lesions in ovarian cancer is not fully described by a mono-exponential function [10] and more advanced models potentially provide a better description of the data, which would enable more detailed investigation of differences between tumour sites. A good fit to the data using a minimum number of parameters and good repeatability are key requirements of such models.

An example of a model with a minimum number of additional parameters is the stretched exponential model, which only utilises one additional parameter $(\alpha)$, to describe the deviation of the decay curve from a mono-exponential curve (Eq. 2). The distributed diffusion coefficient (DDC) is conventionally used in the exponent [11].

$S(b)=S_{0} \exp \left(-(b . D D C)^{\alpha}\right)$

The stretched exponential model has been used preclinically in healthy rat brains [11] and a rat model of glioma [12] and has also been applied clinically in high-grade glioma [13] and prostate cancer [14]. Stretched exponential behaviour may arise from a distribution of mono-exponential decay processes, for example, multiple pools of water molecules, with different diffusion coefficients within each voxel [11]. A stretched exponential description of the DW-MRI signal can also be derived from a generalisation of the Bloch-Torrey equation using fractional order space and time derivatives to describe a distribution of jump lengths or jump waiting-times in a random walk [15].

Another model, the bi-exponential model, also called intravoxel incoherent motion (IVIM), describes two distinct components in the signal decay (Eq. 3) [16]. The biexponential model has been shown to describe the DW-MRI signal in prostate [17], liver [18], and kidneys [19]. The fast component $\left(D^{*}\right)$ is associated with the signal decay due to perfusion and the slow component (D) is associated with diffusion processes in the sample. The perfusion fraction is described by the parameter $f$ in Eq. 3 .

$S(b)=S_{0}\left(f \exp \left(-b D^{*}\right)+(1-f) \exp (-b D)\right)$

We therefore aimed to assess repeatability and goodnessof-fit of parameters measured from mono-exponential (ADC), stretched exponential (DDC, $\alpha$ ), and bi-exponential (D, $\left.f, \mathrm{D}^{*}\right)$ models in data from ovarian cancer localised in ovary, omentum, peritoneum, and lymph nodes, before and after treatment, in order to determine the best model for future treatment response trials using DW-MRI in this disease. A formal evaluation of response is outside the remit of the current work, which only seeks to establish the optimal model for analysis of pre-treatment and post-treatment data at multiple metastatic sites in ovarian cancer.

\section{Materials and methods}

Patients Patients with high-grade ovarian cancer (primary $n=$ 17 , relapsed $n=14$ ) with at least one lesion larger than $2 \mathrm{~cm}$ recruited from two institutions as part of an ongoing prospective multicentre clinical trial (DISCOVAR, CCR3694) were studied. Patients with primary disease were scanned twice before starting chemotherapy while those with relapsed disease were scanned only once. The median interval between two pre-treatment scans was 3 days (range 0 days to 7 days). All patients had a scan after three cycles of platinum-based chemotherapy.

Imaging protocol Hyoscine butylbromide (20 mg) i.m. was administered before scanning to reduce image artefacts due to peristalsis. $\mathrm{T}_{1}$-weighted and $\mathrm{T}_{2}$-weighted images were acquired in order to provide anatomical information. A DW sequence with ten diffusion-weightings $(0,50,100,150$, $200,250,300,500,700,900 \mathrm{~s} \mathrm{~mm}^{-2}$ ) was acquired for comprehensive analysis of non-mono-exponential models at a single station as time constraints prevented full volume coverage. The imaging volume for this sequence was positioned on the largest lesion on the baseline examination and copied to the same position on subsequent examinations. The protocols for the DW-MRI sequences are described in Table 1. Stacked DW sequences using four b-values $(0,100,500$, $900 \mathrm{~s} \mathrm{~mm}^{-2}$ ) acquired in three stations covering the abdomen and pelvis from the symphysis pubis to the top of the diaphragm for response assessment within the trial did not form part of this study.

Analysis Diffusion-weighted images from the additional station (ten b-values between 0 and $900 \mathrm{~s} \mathrm{~mm}^{-2}$ ), positioned on the largest lesion, were analysed in this methodological study. Smaller lesions encompassed by this station were also 
Table 1 Protocols used for DW-MRI on two scanners

\begin{tabular}{|c|c|c|}
\hline & Scanner 1 & Scanner 2 \\
\hline Scanner & Siemens MAGNETOM Avanto $1.5 \mathrm{~T}$ & GE Discovery $1.5 \mathrm{~T}$ \\
\hline Receive coil & $\begin{array}{l}\text { anterior body matrix and } \\
\text { posterior spine matrix }\end{array}$ & 32 channel body array \\
\hline Slice orientation & axial & axial \\
\hline Breathing & free breathing & free breathing \\
\hline Sequence & single-shot EPI & single-shot EPI \\
\hline Averages & 4 & 4 \\
\hline Field-of-view / mm (read) $\times$ mm (phase) & $380 \times 332$ & $380 \times 334$ \\
\hline Acquired matrix (read) & 128 & 128 \\
\hline Reconstructed matrix & 256 & 256 \\
\hline Acquired pixel size $/ \mathrm{mm} \times \mathrm{mm}$ & $3 \times 3$ & $3 \times 3$ \\
\hline Slice thickness / mm & 6 & 6 \\
\hline Number of slices & 26 & 26 \\
\hline Parallel imaging & $\begin{array}{l}\text { GRAPPA (reduction factor } \\
2 ; 36 \text { ACS lines) }\end{array}$ & ASSET (reduction factor 2) \\
\hline PE direction & $\mathrm{AP}$ & $\mathrm{AP}$ \\
\hline Receive bandwidth & $1776 \mathrm{~Hz} /$ pixel & $\pm 125 \mathrm{kHz}(1953 \mathrm{~Hz} /$ pixel $)$ \\
\hline $\mathrm{TR} / \mathrm{ms}$ & 8000 & 8000 \\
\hline $\mathrm{TE} / \mathrm{ms}$ & 75 & 81 \\
\hline Fat suppression & SPAIR & water-selective excitation \\
\hline Diffusion gradient scheme & bipolar & DSE \\
\hline Diffusion encoding scheme & three-scan trace & ALL \\
\hline $\begin{array}{l}\text { Diffusion weightings (b-values) } \\
\text { for full volume coverage } / \mathrm{s} \mathrm{mm}^{-2}\end{array}$ & $0,100,500,900$ & $0,100,500,900$ \\
\hline $\begin{array}{l}\text { Diffusion weightings (b-values) for } \\
\text { additional station } / \mathrm{s} \mathrm{mm}^{-2}\end{array}$ & $\begin{array}{l}0,50,100,150,200,250,300 \\
\quad 500,700,900\end{array}$ & $\begin{array}{l}0,50,100,150,200,250 \\
\quad 300,500,700,900\end{array}$ \\
\hline
\end{tabular}

included in the analysis. Regions of interest (ROIs) were drawn by region growing on computed DW images $(b=$ $1000 \mathrm{~s} \mathrm{~mm}^{-2}$ ) using in-house software (Adept, Institute of Cancer Research, London) [20]. Computed DW images with high b-values have been shown to have better signal-to-noise ratio (SNR) than acquired images at the same b-value and provide improved conspicuity of lesions compared with acquired images at lower b-values [20]. The use of computed DW images also ensures that the effects of motion between images of different b-values are taken into account in the ROI. In all cases the ROIs were drawn with reference to the anatomical $\mathrm{T}_{1}$-weighted and $\mathrm{T}_{2}$-weighted images. ROIs were drawn on every slice on which the lesion appeared (between two and 23 slices). Mono-exponential, stretched exponential and bi-exponential curves (Eqs. 1, 2, and 3, respectively) were fitted to all ten $b$-values for each pixel in the lesion using a least-squares fit (trust-region-reflective algorithm, Matlab 2013, MathWorks Inc., Natick, MA). When fitting the biexponential curves, starting values of $\mathrm{D}, f$, and $\mathrm{D}^{*}$ were determined from a least-squares fit of a mono-exponential curve to the signal at the lowest five b-values and another mono-exponential curve fitted to the remaining signal at the higher b-values. These starting values were used for the leastsquares fit of the bi-exponential curve to the data at all ten b- values. The goodness-of-fit of the mono-exponential, stretched exponential, and bi-exponential models were compared using the Akaike information criterion (AIC), which imposes a penalty for additional parameters in the model [21]. In order to reduce the sensitivity to outlier values, the median value of each fitted parameter from all pixels in the lesion was used for analysis of repeatability.

The repeatability of estimates of $\mathrm{ADC}, \mathrm{DDC}, \alpha, \mathrm{D}, f$, and $\mathrm{D}^{*}$ were assessed using pairs of pre-treatment measurements from 22 lesions from patients with primary disease using the method of Bland and Altman [22]. Bland-Altman plots of untransformed data showed a relationship between the differences in the repeated measurements and their means that was improved by using the natural logarithm of the data [22]. The coefficient of variation $(\mathrm{CV})$ of the log-transformed data was used to describe the repeatability of the fitted parameters $(\mathrm{CV}=$ $\sqrt{ }\left[\exp \left(\Sigma \mathrm{d}^{2} / 2 \mathrm{~N}\right)-1\right]$, where $\Sigma \mathrm{d}^{2}$ is the sum of squared differences between pairs of measurements and $\mathrm{N}$ is the number of lesions).

\section{Results}

Thirty-nine lesions were evaluated prior to treatment in newly diagnosed or relapsed ovarian cancer, comprising nine ovarian 
lesions, 11 omental lesions, 16 peritoneal lesions, and three lymph nodes. Additionally, 31 lesions imaged after three cycles of treatment (seven ovarian lesions, eight omental lesions, 14 peritoneal lesions, and two lymph nodes) were included in the statistical evaluation to assess goodness-of-fit of the model post-treatment. Eight lesions were only included in pre-treatment analysis as three patients (a total of five lesions) withdrew from the study before post-treatment scans and a further three lesions were not visible on post-treatment scans. The numbers of pixels per lesion ranged from 42 to 136,924 (median 4580, interquartile range 21,340).

Tumours exhibited high signal intensities on trace diffusion-weighted images (Fig. 1a) and low values on corresponding ADC, DDC, and D maps (Fig. 1b-g). Lesions from all tumour sites (ovarian lesions, omental lesions, peritoneal lesions, and lymph nodes) were well depicted on high b-value images, as described in previous studies, which showed that DW-MRI aids detection and delineation of lesions [4].

An example of mono-exponential, stretched exponential, and bi-exponential curves fitted to the data from one pixel in an ovarian lesion in a pre-treatment scan is illustrated in Fig. 2. The fitted parameters for the lesion were: $\mathrm{ADC}=1.35 \times 10^{-3} \mathrm{~mm}^{2} \mathrm{~s}^{-1}$, $\mathrm{DDC}=1.33 \times 10^{-3} \mathrm{~mm}^{2} \mathrm{~s}^{-1}, \alpha=0.76, \mathrm{D}=1.14 \times 10^{-3} \mathrm{~mm}^{2} \mathrm{~s}^{-1}, f=$ 0.14 , and $\mathrm{D}^{*}=1.41 \times 10^{-2} \mathrm{~mm}^{2} \mathrm{~s}^{-1}$. The values of AIC were 29.5, 1.6, and 10.2 for the mono-exponential, stretched exponential, and bi-exponential models, respectively, indicating that the stretched exponential model is the preferred model in this instance.

Repeatability estimates for each of the models from repeated baseline measurements indicated CVs in the range $3 \%$ to $4 \%$ for $\mathrm{ADC}$ and $\mathrm{DDC}$, rising to $7 \%$ for $\alpha$, with very large $\mathrm{CVs}$ for $\mathrm{D}, f$, and $\mathrm{D}^{*}$. These data are listed in Table 2 and illustrated with Bland-Altman plots (Fig. 3). After excluding seven lesions which, by inspection of the Bland-Altman plot, were considered

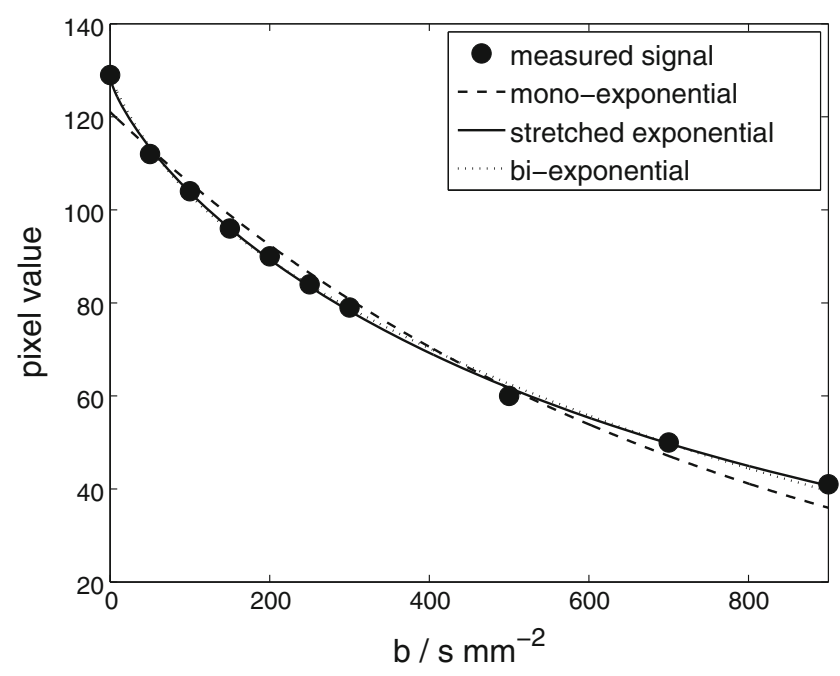

Fig. 2 Mono-exponential, stretched exponential and bi-exponential curves fitted to one pixel in an ovarian lesion measured in a pretreatment scan

to be outliers due to very large differences $(>50 \%)$ in $\mathrm{D}^{*}$ between baseline measurements, the $\mathrm{CV}$ of $\mathrm{D}, f$, and $\mathrm{D}^{*}$ were reduced to $6.4 \%, 18.2 \%$, and $13.6 \%$, respectively.

Owing to the poor repeatability of $f$ and $\mathrm{D}^{*}$, the biexponential model was excluded from the AIC analysis. The percentages of lesions where the AIC indicated the monoexponential and stretched exponential models were preferred by the largest number of pixels in the lesion in pre-treatment measurements were $18 \%$ and $64 \%$, respectively (Table 3 ). In the remaining $18 \%$ of lesions, the majority of pixels preferred a mono-exponential curve on one pre-treatment scan but a stretched exponential curve on the other scan.

When separated by tumour site (ovarian, omental, peritoneal, and lymph node), the stretched exponential model provided the better fit to the majority of pixels in the largest number of

\section{(a) trace image, $\mathrm{b}=900 \mathrm{~s} \mathrm{~mm}^{-2}$}

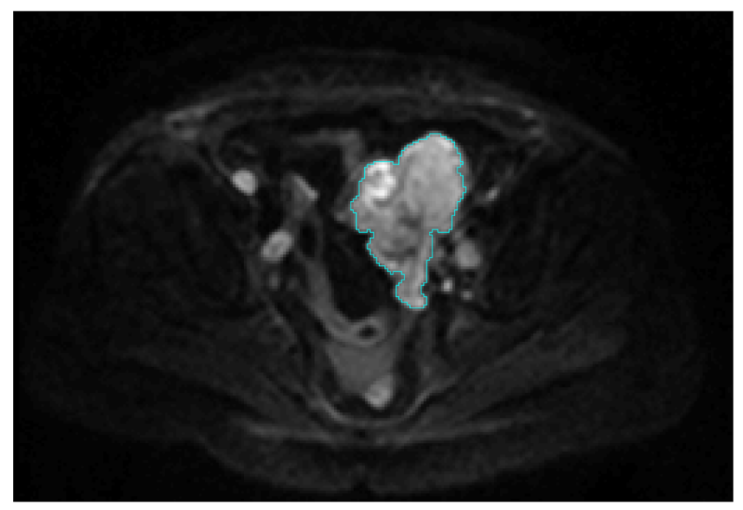

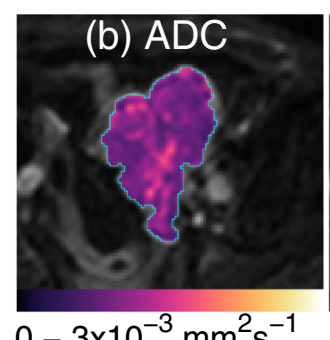

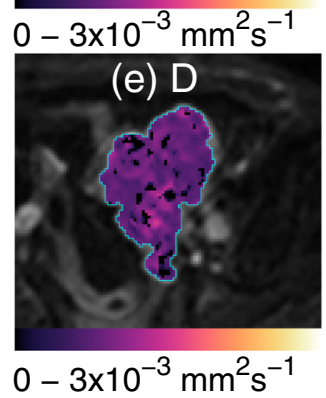

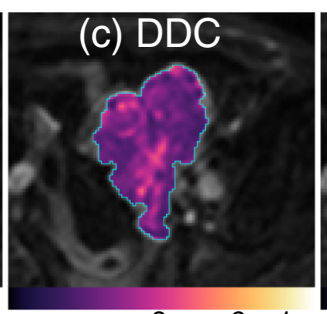
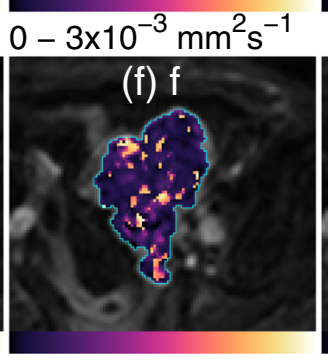

$0-1$
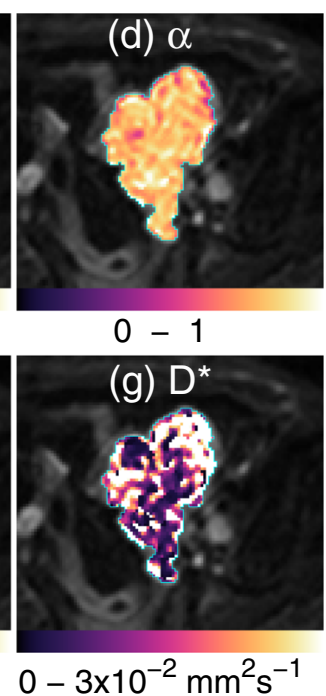

Fig. 1 (a) Diffusion-weighted trace image $\left(b=900 \mathrm{~s} \mathrm{~mm}^{-2}\right)$ of the central slice of an ovarian lesion. (b) ADC estimates from fitting mono-exponential model in ROI. (c, d) DDC and $\alpha$ from the stretched exponential model. $(\mathbf{e}, \mathbf{f}, \mathbf{g}) \mathrm{D}, f$, and $\mathrm{D}^{*}$ from the bi-exponential model 
Table 2 Coefficients of Variation of parameters derived from monoexponential (ADC), stretched exponential (DDC, $\alpha$ ) and biexponential $\left(\mathrm{D}, f, \mathrm{D}^{*}\right)$ models in pre-treatment data in patients with primary disease

\begin{tabular}{ll}
\hline Parameter & $\begin{array}{l}\text { Coefficient of } \\
\text { Variation (\%) }\end{array}$ \\
\hline ADC & 3.1 \\
DDC & 4.3 \\
$\alpha$ & 7.0 \\
$\mathrm{D}$ & 13.2 \\
$f$ & 44.0 \\
$\mathrm{D}^{*}$ & 165.1 \\
\hline
\end{tabular}

lesions in all sites (Table 4). In seven lesions in patients with primary disease (three ovarian, four omental) the majority of pixels was on a mono-exponential curve on one pre-treatment scan but a stretched exponential curve on the other scan.

Correlation of pre-treatment estimates of DDC with ADC and $\alpha$ with DDC for all 39 lesions studied showed that the estimates of ADC and DDC lay close to the line of identity (solid line) for all lesions $\left(r^{2}=0.997\right)$ (Fig. 4a). However, there was no correlation between $\alpha$ and DDC ( $\left.r^{2}=0.003\right)$ (Fig. 4b).

\section{Discussion}

This study shows that the stretched exponential model provides a better characterisation of DW-MRI data from primary

Fig. 3 Bland-Altman plots on log-log scale (a) ADC from the mono-exponential model, $(\mathbf{b}, \mathbf{c})$ DDC and $\alpha$ from the stretched exponential model (d, e, f) D, $f$, and $\mathrm{D}^{*}$ from the bi-exponential model. Solid lines show mean difference between two estimates. Dashed lines show $95 \%$ Limits of Agreement. The same scales have been used on the y-axes of graphs (a-d) and (e-f) to aid comparison

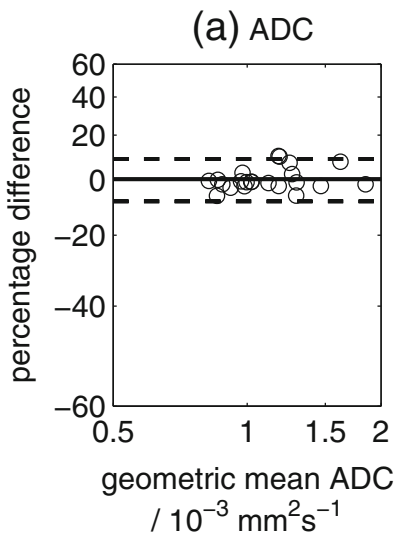

(d) D

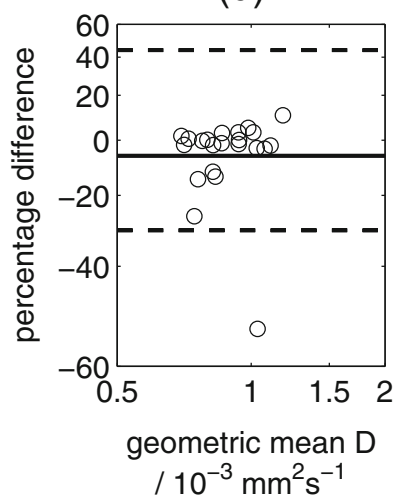

Table 3 Percentage of lesions where mono-exponential and stretched exponential models were preferred by the largest numbers of pixels, as a percentage of all lesions studied at the relevant time-points. Figures in brackets show the numbers of lesions where each model was preferred

\begin{tabular}{lll}
\hline Model & $\begin{array}{l}\text { \% of all lesions } \\
\text { (number) before } \\
\text { treatment where } \\
\text { model preferred }\end{array}$ & $\begin{array}{l}\text { \% of all lesions } \\
\text { (number) after } 3 \text { cycles } \\
\text { of treatment where } \\
\text { models preferred }\end{array}$ \\
\hline Mono-exponential & $18(7)$ & $35(11)$ \\
Stretched exponential & $64(25)$ & $65(20)$ \\
No overall preference $^{\mathrm{a}}$ & $18(7)$ & $\mathrm{n} / \mathrm{a}$
\end{tabular}

${ }^{\mathrm{a}}$ In seven lesions the majority of pixels preferred a mono-exponential curve on one pre-treatment scan but a stretched exponential curve on the other pre-treatment scan

and metastatic ovarian cancer than a mono-exponential or biexponential model. Also, as indicated by the AIC statistics, the preference for the stretched exponential model held true for lesions from different sites (ovarian lesions, omental lesions, peritoneal lesions, and lymph nodes) regardless of whether or not treatment had been given. In addition, the repeatability of the stretched-exponential parameters at baseline was good, whereas that for the bi-exponential was poor. This is a key consideration when applying advanced models that provide a better description of the experimental data because their utility in the investigation of treatment effects or inter-lesion heterogeneity may be
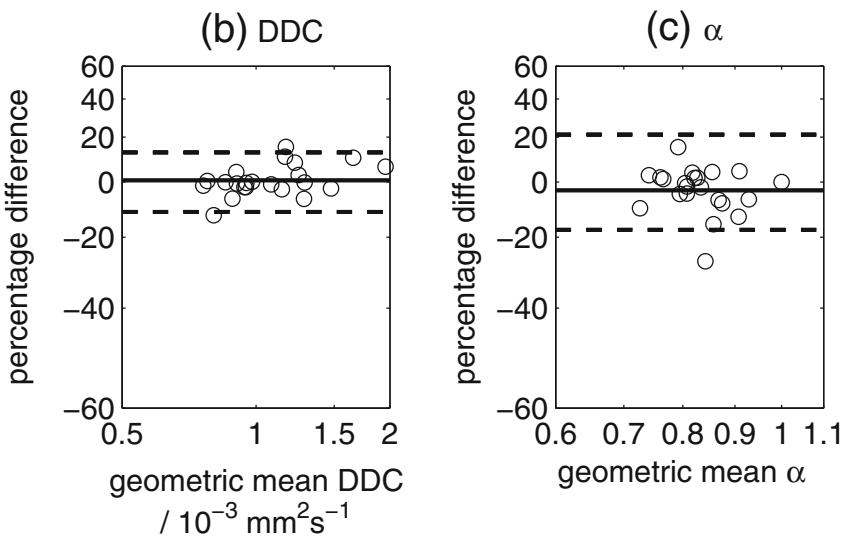

(e) $f$
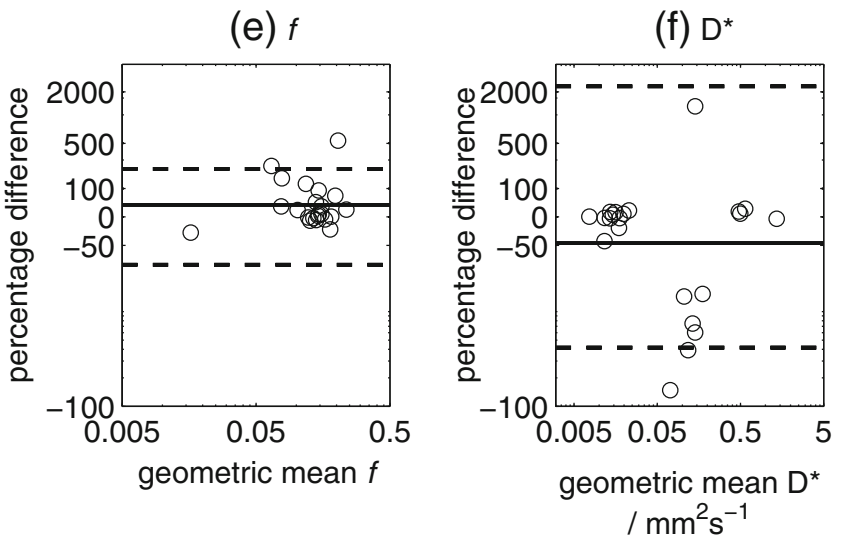
Table 4 Percentage of lesions where mono-exponential and stretched exponential models had the largest numbers of pixels, grouped by tumour site (ovarian, omental, peritoneal, and lymph node). Figures in brackets show the numbers of lesions where each model was preferred

${ }^{\mathrm{a}}$ In three ovarian and four omental lesions the majority of pixels were on a monoexponential curve on one pretreatment scan but a stretched exponential curve on the other pre-treatment scan

\begin{tabular}{llll}
\hline Tumour site & Model & $\begin{array}{l}\text { \% of site-specific lesions } \\
\text { (number) before treatment } \\
\text { where model preferred }\end{array}$ & $\begin{array}{l}\text { \% of site-specific lesions (number) } \\
\text { after 3 cycles of treatment where } \\
\text { model preferred }\end{array}$ \\
\hline Ovarian & Mono-exponential & $0(0)$ & $43(3)$ \\
& Stretched exponential $^{\mathrm{a}}$ & $67(6)$ & $57(4)$ \\
& No overall preference $^{\mathrm{a}}$ & $33(3)$ & $\mathrm{n} / \mathrm{a}$ \\
Omental & Mono-exponential & $18(2)$ & $25(2)$ \\
& Stretched exponential & $45(5)$ & $75(6)$ \\
& No overall preference & $36(4)$ & $\mathrm{n} / \mathrm{a}$ \\
Peritoneal & Mono-exponential & $31(5)$ & $43(6)$ \\
& Stretched exponential & $69(11)$ & $57(8)$ \\
& No overall preference & $\mathrm{n} / \mathrm{a}$ \\
Lymph node & Mono-exponential & $0(0)$ & $0(0)$ \\
& Stretched exponential & $100(3)$ & $100(2)$ \\
& No overall preference & $\mathrm{n} / \mathrm{a}$ & $\mathrm{n} / \mathrm{a}$ \\
\hline
\end{tabular}

undermined if they have poor repeatability. The very good repeatability $(3.1 \%)$ of the ADC estimates from the monoexponential model is in agreement with results of other studies [7, 23-25]. The stretched exponential model has comparable repeatability to the mono-exponential model $(4.3 \%$ for DDC; $7.0 \%$ for $\alpha$ ) indicating that use of this model does not compromise measurement repeatability. The bi-exponential model was unsuitable for analysis of these data owing to its poor repeatability, particularly for $f$ and $\mathrm{D}^{*}$. The poor repeatability of $f$ and D* indicate that the bi-exponential model may be more sensitive to noise in the data than the other models as has been indicated by previous studies $[14,26,27]$ with similarly poor repeatability of $f$ and $\mathrm{D}^{*}$ [25]. The outliers, defined here as differences larger than $50 \%$ between two baseline measurements of $\mathrm{D}^{*}$, may also reflect the sensitivity of the bi-exponential model to noise in the data. Although the repeatability can be improved by excluding outliers, this was only possible in this study because repeat baseline measurements were acquired. Where a treatment effect is being studied, it may be impossible to distinguish between an outlier and a large treatment effect for a given individual.

The stretched exponential model was the most efficient of the non-mono-exponential models as it only involves estimation of one additional parameter. As demonstrated by the repeatability of $\mathrm{D}, f$, and $\mathrm{D}^{*}$, more advanced diffusion models risk over-fitting the data and increasing the sensitivity to noise, particularly in extra-cranial applications. There is also a
Fig. 4 Plots of pre-treatment estimates of (a) DDC against ADC and (b) $\alpha$ against DDC for all lesions. Solid line in (a) shows line of identity (a)

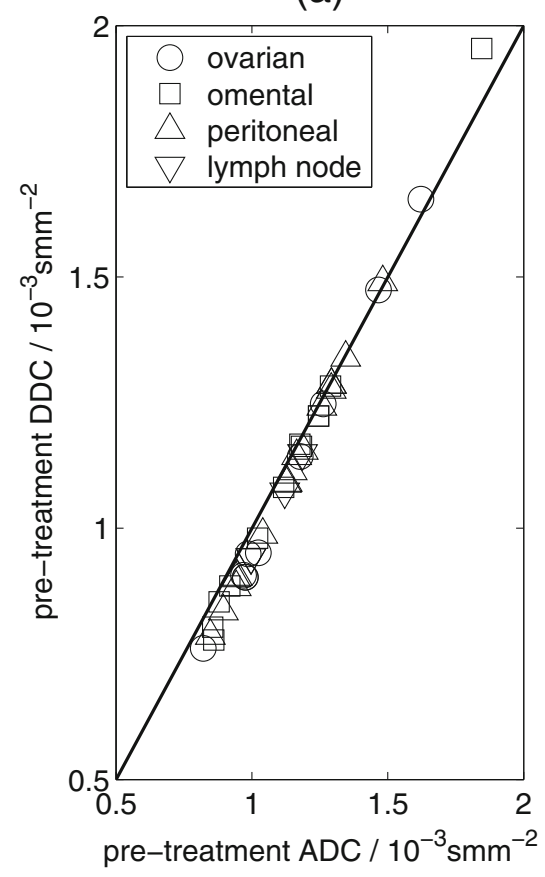

(b)

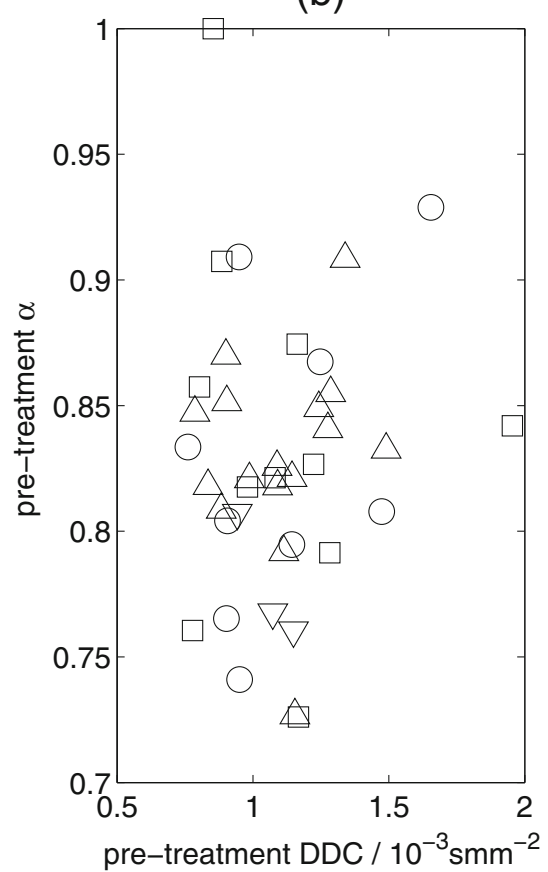


practical argument for choosing an efficient model because the number of b-values acquired must be at least as large as the number of parameters in the model, potentially leading to very long acquisition times for the data required to support more advanced models. Moreover, since $\alpha$ is not correlated with DDC any information it provides will be complementary to DDC, and so it may provide a means of assessing inter-lesion heterogeneity at baseline or differential response to treatment in a larger cohort of patients. The strong correlation of DDC with ADC indicates that DDC provides similar information to ADC.

An apparent limitation of this study was that the lower $\mathrm{b}$-values used in this sequence (lower four b-values 0 , $50,100,150 \mathrm{~s} \mathrm{~mm}^{-2}$ ) may not provide sufficient data to support estimation of the fast component of a biexponential model, in particular $\mathrm{D}^{*}$. The number of $\mathrm{b}$ values and their values at low diffusion-weightings was limited by the requirement for a common sequence across a wide range of clinical platforms for implementation in a multicentre trial. However, the bi-exponential model is typically used to characterise data which exhibit a steep deviation from mono-exponential behaviour at low diffusion-weightings, which is not the case in these data where the deviation from mono-exponential behaviour is observed at all diffusion-weightings (Fig. 2).

In summary, a stretched exponential model provides a good fit to DW-MRI data from ovarian lesions, omental lesions, peritoneal lesions, and lymph nodes in pretreatment and post-treatment measurements. It has comparable repeatability to the mono-exponential model and provides a better description of the data in the majority of lesions both before and after treatment. The extra parameter from the stretched exponential model provides scope for further investigation of inter-lesion heterogeneity or response to treatment.

Acknowledgments The scientific guarantor of this publication is Professor Nandita deSouza, Professor of Translational Imaging, MRI unit, Institute of Cancer Research and Royal Marsden Hospital, Downs Road, Sutton, Surrey. SM2 5PT. UK. The authors of this manuscript declare no relationships with any companies, whose products or services may be related to the subject matter of the article. This study has received funding by CRUK BIDD grant C1353/A12762 and CRUK and EPSRC Cancer Imaging Centre in association with MRC and Department of Health C1060/A10334 and NHS funding to the Clinical Research Facility in Imaging and the NIHR Biomedical Research Centre. We acknowledge the support of the National Institute for Health Research, through the Cancer Research Network (NCRN). No complex statistical methods were necessary for this paper. Institutional Review Board approval was obtained. Written informed consent was obtained from all subjects (patients) in this study. Methodology: prospective, observational, multicentre study.

Open Access This article is distributed under the terms of the Creative Commons Attribution Noncommercial License which permits any noncommercial use, distribution, and reproduction in any medium, provided the original author(s) and the source are credited.

\section{References}

1. Fujii S, Matsusue E, Kanasaki Y et al (2008) Detection of peritoneal dissemination in gynecological malignancy: evaluation by diffusionweighted MR imaging. Eur Radiol 18:18-23

2. Low RN, Sebrechts CP, Barone RM, Muller W (2009) Diffusionweighted MRI of peritoneal tumors: comparison with conventional MRI and surgical and histopathologic findings - a feasibility study. AJR Am J Roentgenol 193:461-470

3. Namimoto T, Awai K, Nakaura T, Yanaga Y, Hirai T, Yamashita Y (2009) Role of diffusion-weighted imaging in the diagnosis of gynecological diseases. Eur Radiol 19:745-760

4. Kyriazi S, Collins DJ, Morgan VA, Giles SL, deSouza NM (2010) Diffusion-weighted imaging of peritoneal disease for noninvasive staging of advanced ovarian cancer. Radiographics 30:1269-1285

5. Sala E, Rockall A, Rangarajan D, Kubik-Huch RA (2010) The role of dynamic contrast-enhanced and diffusion weighted magnetic resonance imaging in the female pelvis. Eur J Radiol 76:367-385

6. Michielsen K, Vergote I, Op de Beeck K et al (2014) Whole-body MRI with diffusion-weighted sequence for staging of patients with suspected ovarian cancer: a clinical feasibility study in comparison to CT and FDG-PET/CT. Eur Radiol 24:889-901

7. Kyriazi S, Collins DJ, Messiou C et al (2011) Metastatic ovarian and primary peritoneal cancer: assessing chemotherapy response with diffusion-weighted MR imaging - value of histogram analysis of apparent diffusion coefficients. Radiology 261:182-192

8. Sala E, Kataoka MY, Priest AN et al (2012) Advanced ovarian cancer: multiparametric MR imaging demonstrates response- and metastasis-specific effects. Radiology 263:149-159

9. Kyriazi S, Collins DJ, Morgan VA, Giles SL, deSouza NM (2010) Diffusion-weighted imaging of ovarian-related peritoneal carcinomatosis: assessment of chemotherapy response in relation to anatomical site. In: Proceedings of the International Society of Magnetic Resonance in Medicine, presentation number 369

10. Sala E, Priest AN, Kataoka M et al (2010) Apparent diffusion coefficient and vascular signal fraction measurements with magnetic resonance imaging: feasibility in metastatic ovarian cancer at 3 Tesla. Eur Radiol 20:491-496

11. Bennett KM, Schmainda KM, Bennett (Tong) R, Rowe DB, Lu H, Hyde JS (2003) Characterization of continuously distributed cortical water diffusion rates with a stretched-exponential model. Magn Reson Med 50:727-734

12. Bennett KM, Hyde JS, Rand SD et al (2004) Intravoxel distribution of DWI decay rates reveals C6 glioma invasion in rat brain. Magn Reson Med 52:994-1004

13. Kwee TC, Galban CG, Tsien C et al (2010) Comparison of apparent diffusion coefficients and distributed diffusion coefficients in highgrade gliomas. J Magn Reson Imaging 31:531-537

14. Mazaheri Y, Afaq A, Rowe DB, Lu Y, Shulka-Dave A, Grover J (2012) Diffusion-weighted magnetic resonance imaging of the prostate: improved robustness with stretched exponential modeling. J Comput Assist Tomogr 36:695-703

15. Magin RL, Abdullah O, Baleanu D, Zhou XJ (2008) Anomalous diffusion expressed through fractional order differential operators in the Bloch-Torrey equation. J Magn Reson 190:255-270

16. Le Bihan D, Breton E, Lallemand D, Aubin M-L, Vignaud J, LavalJeantet M (1988) Separation of diffusion and perfusion in intravoxel incoherent motion MR imaging. Radiology 168:497-505

17. Riches SF, Hawtin K, Charles-Edwards EM, deSouza NM (2009) Diffusion-weighted imaging of the prostate and rectal wall: comparison of biexponential and monoexponential modelled diffusion and associated perfusion coefficients. NMR Biomed 22:318-325

18. Koh D-M, Collins DJ, Orton MR (2011) Intravoxel incoherent motion in body diffusion-weighted MRI: reality and challenges. AJR Am J Roentgenol 196:1351-1361 
19. Zhang JL, Sigmund EE, Rusinek H et al (2012) Optimization of bvalue sampling for diffusion-weighted imaging of the kidney. Magn Reson Med 67:89-97

20. Blackledge MD, Leach MO, Collins DJ, Koh D-M (2011) Computed diffusion-weighted MR imaging may improve tumor detection. Radiology 261:573-581

21. Akaike H (1974) A new look at the statistical model identification. IEEE Trans Autom Control 19:716-723

22. Bland MJ, Altman DG (1986) Statistical methods for assessing agreement between two methods of clinical measurement. Lancet 327:307-310

23. Koh D-M, Blackledge M, Collins DJ et al (2009) Reproducibility and changes in the apparent diffusion coefficients of solid tumours treated with combretastatin A4 phosphate and bevacizumab in a two-centre phase I clinical trial. Eur Radiol 19:2728-2738
24. Messiou C, Collins DJ, Morgan VA, deSouza NM (2011) Optimising diffusion weighted MRI for imaging metastatic and myeloma bone disease and assessing reproducibility. Eur Radiol 21:1713-1718

25. Andreou A, Koh DM, Collins DJ et al (2013) Measurement reproducibility of perfusion fraction and pseudodiffusion coefficient derived by intravoxel incoherent motion diffusion-weighted MR imaging in normal liver and metastases. Eur Radiol 23:428434

26. Le Bihan D, Turner R, Moonen CTW, Pekar J (1991) Imaging of diffusion and microcirculation with gradient sensitization: design, strategy, and significance. J Magn Reson Imaging 1:728

27. Mulkern RV, Haker SJ, Maier SE (2009) On high b diffusion imaging in the human brain: ruminations and experimental insights. Magn Reson Imaging 27:1151-1162 